\title{
2022 Update of the Korean Clinical Practice Guidelines for Stroke: Antithrombotic Therapy for Patients with Acute Ischemic Stroke or Transient Ischemic Attack
}

\author{
Hong-Kyun Park, , Sang-Bae Ko, ${ }^{\mathrm{b}}$ Keun-Hwa Jung, ${ }^{\mathrm{b}}$ Min Uk Jang, ${ }^{\mathrm{c}}$ Dae-Hyun Kim, ${ }^{\mathrm{d}}$ Joon-Tae Kim, ${ }^{\mathrm{e}}$

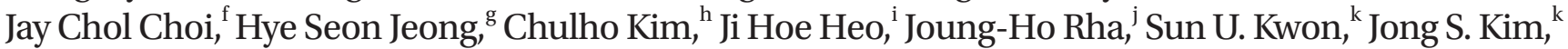 \\ Byung-Chul Lee, ${ }^{1}$ Hee-Joon Bae, ${ }^{\mathrm{m}}$ Byung-Woo Yoon, ${ }^{\mathrm{n}}$ Keun-Sik Hong ${ }^{\mathrm{a}}$ \\ aDepartment of Neurology, Inje University Ilsan Paik Hospital, College of Medicine, Inje University, Goyang, Korea \\ ${ }^{b}$ Department of Neurology, Seoul National University Hospital, Seoul National University College of Medicine, Seoul, Korea \\ 'Department of Neurology, Hallym University Dongtan Sacred Heart Hospital, Hallym University College of Medicine, Hwaseong, Korea \\ ${ }^{d}$ Department of Neurology, Dong-A University Hospital, Dong-A University College of Medicine, Busan, Korea \\ 'Department of Neurology, Chonnam National University Hospital, Chonnam National University Medical School, Gwangju, Korea \\ fDepartment of Neurology, Jeju National University Hospital, Jeju National University School of Medicine, Jeju, Korea \\ ${ }^{9}$ Department of Neurology, Chungnam National University Hospital, Chungnam National University College of Medicine, Daejeon, Korea \\ ${ }^{h}$ Department of Neurology, Hallym University Chuncheon Sacred Heart Hospital, Hallym University College of Medicine, Chuncheon, Korea \\ 'Department of Neurology, Severance Stroke Center, Severance Hospital, Yonsei University College of Medicine, Seoul, Korea \\ 'Department of Neurology, Inha University Hospital, Inha University College of Medicine, Incheon, Korea \\ ${ }^{k}$ Department of Neurology, Asan Medical Center, University of Ulsan College of Medicine, Seoul, Korea \\ 'Department of Neurology, Hallym University Sacred Heart Hospital, Hallym University College of Medicine, Anyang, Korea \\ ${ }^{m}$ Department of Neurology and Cerebrovascular Center, Seoul National University Bundang Hospital, Seoul National University College of \\ Medicine, Seongnam, Korea \\ "Department of Neurology, Uijeongbu Eulji Medical Center, Eulji University, Uijeongbu, Korea
}

Antithrombotic therapy is a cornerstone of acute ischemic stroke (AIS) management and secondary stroke prevention. Since the first version of the Korean Clinical Practice Guideline (CPG) for stroke was issued in 2009, significant progress has been made in antithrombotic therapy for patients with AIS, including dual antiplatelet therapy in acute minor ischemic stroke or high-risk transient ischemic stroke and early oral anticoagulation in AIS with atrial fibrillation. The evidence is widely accepted by stroke experts and has changed clinical practice. Accordingly, the CPG Committee of the Korean Stroke Society (KSS) decided to update the Korean Stroke CPG for antithrombotic therapy for AIS. The writing members of the CPG committee of the KSS reviewed recent evidence, including clinical trials and relevant literature, and revised recommendations. A total of 35 experts were invited from the KSS to reach a consensus on the revised recommendations. The current guideline update aims to assist healthcare providers in making well-informed decisions and improving the quality of acute stroke care. However, the ultimate treatment decision should be made using a holistic approach, considering the specific medical conditions of individual patients.
Correspondence: Keun-Sik Hong Department of Neurology, Inje University Ilsan Paik Hospital, College of Medicine, Inje University, 170 Juhwa-ro, Ilsanseogu, Goyang 10380, Korea Tel: +82-31-910-7680

Fax: +82-31-913-7368 E-mail: nrhks455@gmail.com https://orcid.org/0000-0002-4684-6111

Received: July 23, 2021

Revised: November 4, 2021

Accepted: November 4, 2021

Keywords Acute ischemic stroke; Antithrombotic therapy; Guideline

Copyright (C) 2022 Korean Stroke Society

This is an Open Access article distributed under the terms of the Creative Commons Attribution Non-Commercial License (http://creativecommons.org/licenses/by-nc/4.0/) which permits unrestricted non-commercial use, distribution, and reproduction in any medium, provided the original work is properly cited. 


\section{Introduction}

Previously, aspirin was the only antithrombotic therapy with a proven efficacy for preventing early recurrent stroke and death or dependency in patients with acute ischemic stroke (AIS) in large clinical trials. ${ }^{1-3}$ Major international guidelines and the previous Clinical Practice Guideline (CPG) of the Korean Stroke Society (KSS) recommended aspirin monotherapy as an acute antithrombotic therapy. However, the effect of aspirin in AIS is modest; therefore, large clinical trials have investigated more effective antithrombotic regimens. In 2013, the Clopidogrel in High-Risk Patients with Acute Nondisabling Cerebrovascular Events (CHANCE) trial demonstrated for the first time the greater benefit of dual antiplatelet therapy (DAPT) with clopidogrel plus aspirin over aspirin monotherapy in Chinese patients with minor AIS or high-risk transient ischemic attack (TIA). ${ }^{4}$ The Platelet-Oriented Inhibition in New TIA and Minor Ischemic Stroke (POINT) trial later replicated and reinforced the CHANCE trial results in a broader ethnic population. ${ }^{5}$ Currently, DAPT with clopidogrel plus aspirin is recognized and recommended as a standard management for patients with minor AIS or highrisk TIA. ${ }^{6-8}$ Triple antiplatelet therapies and ticagrelor monotherapy have been investigated in clinical trials, but these regimens were not more effective than standard antiplatelet therapy., ${ }^{9,10}$

Long-term oral anticoagulation with non-vitamin $\mathrm{K}$ antagonist (NOAC) or warfarin is strongly recommended for secondary stroke prevention in patients with atrial fibrillation (AF) and ischemic stroke or TIA." However, there are no clinical trial data regarding the optimal timing to initiate oral anticoagulation during the acute phase when both the risks of recurrent ischemic stroke and hemorrhagic transformation (HT) are high. Nevertheless, oral anticoagulation in patients with AIS with AF is widely used in real-world practice. Recently, based on observational studies and expert consensus, several guidelines have updated their recommendations for oral anticoagulation in AIS and $\mathrm{AF}^{6,12,13}$

To reflect the accumulated evidence, the CPG Committee of the KSS decided to revise the Korean CPG for stroke to provide up-to-date recommendations for antithrombotic therapy in patients with AIS or TIA. The purpose of the current guideline update is to help make well-informed decisions and improve the quality of care for antithrombotic management. The ultimate treatment decision must be made by responsible healthcare providers, patients, and/or their caregivers.

\section{Previous guidelines}

The previous 2009 Korean CPG for antiplatelet therapy and the 2014 Korean CPG for anticoagulant therapy provided the fol- lowing recommendations for patients with AIS or TIA. ${ }^{14}$

\section{Acute stroke management}

\subsection{Acute treatment}

\subsubsection{Antiplatelet agents}

1. In the hemorrhage-excluded, AIS patients, the oral administration of aspirin should start within 24 to 48 hours of onset (the loading dose 160 to $300 \mathrm{mg}$ ) (level of evidence [LOE]: la, grade of recommendation [GOR]: A).

2. Aspirin cannot replace acute interventions including intravenous thrombolysis (LOE: Ia, GOR: A).

3. Aspirin should not be taken within 24 hours of thrombolysis (LOE: la, GOR: A).

4. Intravenous injection of the glycoprotein IIb/IIla receptor antagonists, including abciximab, is not recommended in patients with AIS (LOE: Ib, GOR: A).

\subsubsection{Anticoagulants}

1. There is no scientific evidence of the usefulness of heparin used within 48 hours of ischemic cerebral infarction. It might increase the risk of bleeding, compared with aspirin (LOE: la, GOR: A).

2. Low molecular weight heparin or heparinoids is not recommended as an early treatment of cerebral infarction (LOE: la, GOR: A).

3. Use of anticoagulants within 24 hours of recombinant tissue plasminogen activator administration is not recommended (LOE: Ila, GOR: B).

\section{Methodology}

\section{Process of updating the Korean CPG for antithrombotic therapy in AIS or TIA}

In May 2019, the Guideline Oversight Committee of the KSS decided to update the Korean CPG for antithrombotic therapy in patients with AIS or TIA and appointed members of the writing group recommended by the CPG Committee of the KSS. To achieve consensus, the CPG Committee organized an expert panel consisting of 35 experts. The writing group prepared revised recommendations, and expert panel members reached a consensus after two Delphi rounds in May 2020. The Guideline Oversight Committee reviewed and approved drafts prepared by the writing group.

\section{Evidence search and summary}

For the updated information on antithrombotic therapy in AIS 
Table 1. Level of evidence and grade of recommendation

\begin{tabular}{ll}
\hline Level of evidence (LOE) & Evidence obtained from meta-analysis of randomized controlled trials \\
la & Evidence obtained from at least one randomized controlled trial \\
Ib & Evidence obtained from at least one well-designed controlled study without randomization \\
Ila & Evidence obtained from at least one other type of well-designed quasi-experimental study \\
IIb & Evidence obtained from well-designed non-experimental descriptive studies, such as comparative studies, correlation \\
III & studies and case studies \\
IV & Evidence obtained from expert committee reports or opinions and/or clinical experiences of respected authorities \\
Grade of recommendation (GOR) & Required: at least one randomized controlled trial as part of the body of literature of overall good quality and \\
A (LOE la, Ib) & consistency addressing specific recommendation \\
B (LOE Ila, IIb, III) & Required: availability of well conducted clinical studies but no randomized clinical trials on the topic of \\
C (LOE IV) & Recommendation \\
GPP (good practice points) & Recommended best practice based on the clinical experience of the guideline development group
\end{tabular}

or TIA, we searched and assessed the results of randomized controlled trials (RCTs), pooled analyses, and updated major international guidelines published between January 2009 and May 2020. Additionally, we reviewed relevant articles solicited by experts who participated in the current guideline update.

\section{LOE and GORs}

For each recommendation, the LOE and GOR were determined based on the US Agency for Healthcare Policy and Research (currently the Agency for Healthcare Research and Quality) criteria (Table 1). ${ }^{15}$ We used this grading system to accord with recommendations of other subjects in the Korean CPGs for stroke.

\section{Evidence summary}

\section{Dual antiplatelet therapy with clopidogrel and aspirin}

\section{CHANCE trial}

The CHANCE trial randomized 5,170 Chinese patients with acute minor ischemic stroke (National Institutes of Health Stroke Scale [NIHSS] score $\leq 3)$ or high-risk TIA $\left(\mathrm{ABCD}_{2}\right.$ score $\geq 4$ ) within 24 hours of symptom onset to combination therapy with clopidogrel (300 mg loading on day 1 followed by $75 \mathrm{mg}$ once daily for 90 days) and aspirin (75 to $300 \mathrm{mg}$ on day 1 followed by $75 \mathrm{mg}$ once daily for the first 21 days) or aspirin monotherapy (75 to $300 \mathrm{mg}$ on day 1 followed by $75 \mathrm{mg}$ once daily for 90 days). ${ }^{4}$ The median interval from onset to randomization was 13 hours, and $50 \%$ of patients were enrolled with- in 12 hours of onset. Of the qualifying events, 72\% were AIS.

Compared with the aspirin monotherapy group, the clopidogrel plus aspirin group had a significantly lower risk of the primary efficacy outcome of any recurrent stroke (ischemic or hemorrhagic) at 90 days (8.2\% vs. $11.7 \%$; hazard ratio [HR], 0.68 ; $95 \%$ confidence interval $[\mathrm{Cl}], 0.57$ to $0.81 ; P<0.001$; absolute risk reduction [ARR], 3.5\%; number needed to treat [NNT], 29). The benefit was consistent for all predefined subgroups. Among secondary efficacy outcomes, clopidogrel plus aspirin compared to aspirin monotherapy reduced the risk of any stroke, myocardial infarction, or vascular death (8.4\% vs. $11.9 \%$; $\mathrm{HR}, 0.69 ; 95 \% \mathrm{Cl}, 0.58$ to $0.82 ; P<0.001 ; A R R, 3.5 \%$; NNT, 29) and ischemic stroke (7.9\% vs. $11.4 \% ; \mathrm{HR}, 0.67 ; 95 \% \mathrm{Cl}, 0.56$ to $0.82 ; P<0.001 ; A R R, 3.5 \%$; NNT, 29). There were no differences in the risk of moderate-to-severe bleeding ( $0.3 \%$ vs. $0.3 \%$, $P=0.73$ ) or hemorrhagic stroke ( $0.3 \%$ vs. $0.3 \%, P=0.98)$.

\section{POINT trial}

The POINT trial was designed earlier, but was completed later than the CHANCE trial. Similar to the CHANCE trial, the POINT trial enrolled 4,881 patients with acute minor ischemic stroke (NIHSS score $\leq 3)$ or high-risk TIA $\left(\mathrm{ABCD}_{2}\right.$ score $\left.\geq 4\right)$ from 10 countries in North America, Europe, Australia, and New Zealand. In contrast to the CHANCE trial, the POINT trial randomized patients within 12 hours after symptom onset, used a 600-mg loading dose of clopidogrel and 90 days of clopidogrel plus aspirin in the DAPT group, and enrolled patients with diverse ethnicities (predominantly white). ${ }^{5}$ The primary efficacy outcome was a composite of ischemic stroke, myocardial infarction, or death from ischemic vascular causes. At the time of 
enrolling $83.6 \%$ of the planned number of patients, the POINT trial was terminated early because of the excess of major hemorrhage beyond the prespecified boundary in the DAPT group, and efficacy analysis was conducted. The median interval from onset to randomization was 7.4 hours, and $32 \%$ of patients were enrolled within 6 hours of onset. Of the qualifying events, $57 \%$ were AlSs.

Compared to the aspirin monotherapy group, the DAPT group had a significantly lower risk of the primary efficacy outcome at 90 days ( $5.0 \%$ vs. $6.5 \%$; $\mathrm{HR}, 0.75 ; 95 \% \mathrm{Cl}, 0.59$ to 0.95 ; $P=0.020 ; A R R, 1.5 \%$; NNT, 67). This effect was consistent in all predefined subgroups. Among secondary efficacy outcomes, DAPT compared with aspirin monotherapy reduced the risk of ischemic stroke (4.6\% vs. $6.3 \%$; $\mathrm{HR}, 0.72 ; 95 \% \mathrm{Cl}, 0.56$ to 0.92 ; $P=0.01$; ARR, $1.7 \%$; NNT, 59$)$ and any stroke $(4.8 \%$ vs. $6.4 \%$; $H R, 0.74 ; 95 \% \mathrm{Cl}, 0.58$ to $0.94 ; P=0.01 ; A R R, 1.6 \%$; NNT, 63). However, the incidence of major hemorrhage was significantly higher in the DAPT group than in the aspirin monotherapy group ( $0.9 \%$ vs. $0.4 \% ; \mathrm{HR}, 2.32 ; 95 \% \mathrm{Cl}, 1.10$ to $4.87 ; P=0.02$; absolute hazard increase, $0.5 \%$; number needed to hazard, 200).

In post hoc analysis, the benefit of DAPT over aspirin monotherapy was significant in the first 7 days and in the first 30 days, but disappeared from day 31 to day 90 , while the risk of major hemorrhage with DAPT significantly increased from day 8 to day 90. Accordingly, per 1,000 patients treated, DAPT for 90 days compared to aspirin monotherapy would prevent 15 major ischemic events and cause five additional major hemorrhages, and DAPT for 30 days would prevent 19 major ischemic events and cause two additional major hemorrhages.

\section{Pooled analysis and meta-analysis}

In a pooled individual patient-level data analysis of the CHANCE and POINT trials $(n=10,051)$, compared with aspirin monotherapy, clopidogrel plus aspirin significantly reduced the risk of major ischemic events (ischemic stroke, myocardial infarction, or ischemic vascular death) (6.5\% vs. $9.1 \%$; HR, 0.70 ; $95 \% \mathrm{Cl}, 0.61$ to $0.81 ; P<0.001)$. DAPT also reduced the risk of ischemic stroke (6.3\% vs. $8.9 \%$; $\mathrm{HR}, 0.69 ; 95 \% \mathrm{Cl}, 0.60$ to 0.80 ; $P<0.001)$ and any stroke (6.5\% vs. 9.1\%; HR, $0.71 ; 95 \% \mathrm{Cl}$, 0.61 to $0.81 ; P<0.001)$ compared with aspirin monotherapy. Major hemorrhages ( $0.6 \%$ vs. $0.4 \%$; $\mathrm{HR}, 1.67 ; 95 \% \mathrm{Cl}, 0.93$ to 2.99; $P=0.09)$ and hemorrhagic stroke $(0.3 \%$ vs. $0.2 \% ; H R$, $1.16 ; 95 \% \mathrm{Cl}, 0.52$ to $2.60 ; P=0.71)$ were more frequent with DAPT than with aspirin monotherapy; however, the differences were not significant.

In the time-course analysis, the benefit of DAPT for major ischemic events was largely achieved within the first 21 days
( $5.2 \%$ vs. $7.8 \%$; $\mathrm{HR}, 0.66 ; 95 \% \mathrm{Cl}, 0.56$ to $0.77 ; P<0.001$ ), and it was not significant between 22 and 90 days (1.4\% vs. 1.5\%; $\mathrm{HR}, 0.94 ; 95 \% \mathrm{Cl}, 0.67$ to $1.32 ; P=0.72$ ). For net clinical benefit (major ischemic events prevented-major hemorrhages increased), DAPT had a clear benefit for the first 21 days, but a negligible benefit from day 22 to day $90 .{ }^{16}$

\section{Triple antiplatelet therapy}

The Triple Antiplatelets for Reducing Dependency after Ischaemic Stroke (TARDIS) trial compared triple antiplatelet therapy (aspirin, dipyridamole, and clopidogrel) and standard therapy (clopidogrel monotherapy or aspirin plus dipyridamole) in patients with acute non-cardioembolic ischemic stroke (presenting with limb weakness, dysphasia, or neuroimaging-positive hemianopia) or TIA (presenting with at least 10 minutes of limb weakness or isolated dysphasia) within 48 hours from symptom onset. ${ }^{9}$ Patients from the United Kingdom (95\%), Georgia, Denmark, and New Zealand were randomized to triple therapy $(n=1,556)$ or standard treatment $(n=1,540)$. During the trial, patients maintained their assigned treatment for the first 30 days and were treated according to local guidelines from day 31 to day 90. The TARDIS trial was terminated early after enrollment of 76\% of the planned sample size because of a significant increase in major bleeding events and no signs of efficacy with triple therapy. Of the qualifying events, $72 \%$ were AISs. The median interval from onset to randomization was 29.3 hours, and $69 \%$ of patients were enrolled 24 hours after onset.

There was no difference in the primary efficacy outcome (incidence and severity of recurrent stroke or TIA at 90 days) between the triple and standard therapy groups (6\% vs. $7 \%$; adjusted common odds ratio $[0 R], 0.90 ; 95 \% \mathrm{Cl}, 0.67$ to 1.20 ; $P=0.47)$. However, triple therapy, compared to standard therapy, had significantly more bleeding events (20\% vs. $9 \%)$ and was associated with a worse shift on the five-level ordinal bleeding severity scale (fatal, major, moderate, minor, and none) (adjusted common OR, 2.54; 95\% Cl, 2.05 to 3.16; $P<0.0001)$. Intracranial bleeding occurred more frequently in the triple therapy group (1\% vs. $<1 \% ; \mathrm{HR}, 3.14 ; 95 \% \mathrm{Cl}, 1.14$ to $8.61 ; P=0.026$ ).

\section{Ticagrelor}

The Acute Stroke or Transient Ischemic Attack Treated with Aspirin or Ticagrelor and Patient Outcomes (SOCRATES) trial compared ticagrelor and aspirin in patients with acute minor ischemic stroke (NIHSS score $\leq 5)$ or high-risk TIA $\left(\mathrm{ABCD}_{2}\right.$ score $\left.\geq 4\right)$ within 24 hours of symptom onset..$^{10}$ The ticagrelor group received a 180-mg loading dose followed by $90 \mathrm{mg}$ twice daily for 90 days, and the aspirin group received a 300-mg loading 
dose followed by $100 \mathrm{mg}$ once daily for 90 days. The trial enrolled 13,199 patients from 33 countries (66.5\% white, 26.9\% Asian, $1.8 \%$ black, and $2.1 \%$ other races). Of the enrolled patients, 36.5\% were randomized within 12 hours of onset, and $73.2 \%$ had acute minor ischemic stroke.

Compared to aspirin, ticagrelor did not significantly reduce the primary endpoint of the composite of recurrent stroke, myocardial infarction, or death at 90 days (6.7\% vs. 7.5\%; HR, $0.89 ; 95 \% \mathrm{Cl}, 0.78$ to $1.01 ; P=0.07)$. Of secondary efficacy outcomes, the ticagrelor group, compared to the aspirin group, had lower risks of ischemic stroke (5.8\% vs. $6.7 \%$; HR, 0.87 ; $95 \% \mathrm{Cl}, 0.76$ to $1.00 ; P=0.046)$ and any stroke (5.9\% vs. $6.8 \%$; $H R, 0.86 ; 95 \% \mathrm{Cl}, 0.75$ to $0.99 ; P=0.03)$. However, the predefined statistical plan was a hierarchical testing sequence where the statistical significance for each secondary endpoint could be accepted if the primary endpoint achieved statistical significance. Therefore, the positive results for preventing ischemic stroke and any stroke should be considered exploratory. The ticagrelor and aspirin groups had a similar risk of major bleeding (0.5\% vs. $0.6 \%$; HR, $0.83 ; 95 \% \mathrm{Cl}, 0.52$ to 1.34 ; $P=0.45$ ), but patients on ticagrelor were more likely than those on aspirin to experience dyspnea (6.2\% vs. $1.4 \%$ ).

The Acute Stroke or Transient Ischaemic Attack Treated with Ticagrelor and Acetylsalicylic acid for Prevention of Stroke and Death (THALES) trial was published after completing our consensus achievement for this guideline update; therefore, we have not reflected the results of the THALES trial in the updated recommendations. In brief, the THALES trial compared ticagrelor (180-mg loading dose followed by $90 \mathrm{mg}$ twice daily) plus aspirin (300 to $325 \mathrm{mg}$ on day 1 followed by 75 to $100 \mathrm{mg}$ daily) versus aspirin monotherapy in 11,016 patients with acute minor ischemic stroke (NIHSS score $\leq 5)$ or high-risk TIA $\left(\mathrm{ABCD}_{2}\right.$ score $\geq 6$ or relevant intracranial or extracranial stenosis $\geq 50 \%$ ) within 24 hours of symptom onset. ${ }^{17}$ At 30 days, the ticagrelor plus aspirin group compared to the aspirin monotherapy group had lower risks of the primary efficacy outcome of stroke or death (5.4\% vs. $6.5 \% ; \mathrm{HR}, 0.83 ; 95 \% \mathrm{Cl}, 0.71$ to $0.96 ; P=0.020$ ) and ischemic stroke ( $5.0 \%$ vs. $6.3 \%$; $\mathrm{HR}, 0.79 ; 95 \% \mathrm{Cl}, 0.68$ to 0.93 ; $P=0.004)$. However, the combination therapy group compared to the aspirin group had significantly more cases of severe bleeding (0.5\% vs. $0.1 \% ; \mathrm{HR}, 3.99 ; 95 \% \mathrm{Cl}, 1.74$ to 9.14 ; $P=0.001)$ and intracranial hemorrhage $(\mathrm{ICH})$ or fatal bleeding (0.4\% vs. $0.1 \%$; $\mathrm{HR}, 3.66 ; 95 \% \mathrm{Cl}, 1.48$ to $9.02 ; P=0.005$ ).

\section{Timing of antithrombotic therapy in patients treated with intravenous thrombolysis}

The previous recommendation to avoid antithrombotic therapy within the first 24 hours after intravenous thrombolysis (IVT) was based on expert consensus. No RCT has investigated the timing of antithrombotic initiation after IVT, and data from well-designed observational studies are scarce. A single-center observational study in Korea reported that the early administration of antithrombotics within 24 hours after reperfusion therapy was safe. In this study, of 712 patients treated with reperfusion therapy (34\% IVT only, 34\% IVT plus mechanical thrombectomy [MT], and 32\% MT only), 456 (64\%) patients received antithrombotic therapy within 24 hours after reperfusion therapy. Early initiation of antithrombotic therapy within 24 hours as compared to late initiation after 24 hours did not increase the risk of symptomatic HT (3.3\% vs. 3.1\%; adjusted $\mathrm{OR}, 0.85 ; 95 \% \mathrm{Cl}, 0.35$ to 2.10 ). Any $\mathrm{HT}$ was even lower in the early initiation group (adjusted $\mathrm{OR}, 0.56 ; 95 \% \mathrm{Cl}, 0.35$ to 0.89 ). The rate of 3-month favorable outcomes (modified Rankin Scale [mRS] score 0-1) was comparable between the early and late initiation groups (39.3\% vs. 34.8\%; OR, 1.09; 95\% Cl, 0.75 to 1.59). In the interaction analysis, the modality of reperfusion therapy (IVT, IVT plus MT, or MT) did not modify the associations between the initiation timing and symptomatic $\mathrm{HT}$, any HT, or mRS outcome. ${ }^{18}$ These findings were reflected in the 2019 American Heart Association/American Stroke Association stroke guidelines as follows: Section 3.9. Antiplatelet Treatment-Administration of aspirin is recommended in patients with AIS within 24 to 48 hours after onset. For patients treated with intravenous (IV) alteplase, aspirin administration is generally delayed until 24 hours later, but might be considered in the presence of concomitant conditions for which such treatment given in the absence of IV alteplase is known to provide substantial benefit or withholding such treatment is known to cause substantial risk (class of recommendation: I, LOE: A).

\section{Cilostazol}

The Cilostazol in Acute Ischemic Stroke Treatment (CAIST) trial conducted in Korea was a randomized double-blind non-inferiority trial that compared cilostazol $200 \mathrm{mg} /$ day and aspirin 300 $\mathrm{mg} /$ day in 458 patients with AIS (NIHSS $\leq 15$ ) within 48 hours of symptom onset for 90 days. $^{19}$ The primary endpoint of $90-$ day mRS 0-2 was achieved in 76\% of the cilostazol group and $75 \%$ of the aspirin group. Both intention-to-treat and per-protocol analyses showed non-inferiority of cilostazol to aspirin (one-sided $95 \% \mathrm{Cl}$ of the proportion difference, $-6.15 \%$ to 7.22\%; $P=0.0004$ [intention-to-treat analysis]; one-sided 95\% $\mathrm{Cl}_{1}-9.82 \%$ to $4.51 \% ; P=0.045$ [per-protocol analysis]). Cardiovascular events ( $3 \%$ vs. $4 \%, P=0.41$ ) and bleeding complications ( $11 \%$ vs. $13 \%, P=0.43$ ) were comparable between the two groups. However, the trial had the shortcomings of a small sample size and wide non-inferiority margin. 


\section{Glycoprotein Ilb/IIla receptor antagonists}

An earlier RCT failed to demonstrate the safety and efficacy of IV abciximab in patients with $\mathrm{AIS}^{20}$ Accordingly, the previous Korean stroke guidelines did not recommend IV glycoprotein IIb/IIla receptor antagonists for acute antithrombotic therapy..$^{14}$ In patients treated with MT, rescue therapy is often required for those with incomplete recanalization or re-occlusion due to in situ thrombosis. No RCT has demonstrated the benefit of parenteral glycoprotein Ilb/IIla receptor antagonists as rescue therapy during MT. A recent observational study suggested that tirofiban rescue therapy was safe in patients with unsuccessful recanalization after MT. ${ }^{21}$ The rate of symptomatic ICH was $13.6 \%$ $(21 / 154)$ in patients treated with tirofiban and $16.7 \%$ (80/478) in those who did not receive tirofiban $(\mathrm{OR}, 0.69 ; 95 \% \mathrm{Cl}, 0.36$ to $1.31 ; P=0.26)$. The 90 -day mortality rates in the two groups were $3.2 \%$ and $5.4 \%(\mathrm{OR}, 0.66 ; 95 \% \mathrm{Cl}, 0.36$ to $1.31 ; P=0.15)$. In the subgroup of patients with acute atherosclerotic large artery occlusion, tirofiban use was associated with lower mortality (11.3\% vs. $23.4 \%, P=0.042$ ).

Two recent Korean observational studies reported the safety and feasibility of IV or intra-arterial tirofiban in patients with acute atherosclerotic large artery occlusion who underwent MT. In one study of 118 patients with residual stenosis after MT, the tirofiban group $(n=59)$ had a higher final successful recanalization rate $(81.4 \%$ vs. $42.4 \%, P=0.016)$ and a numerically lower rate of major intracranial bleeding of parenchymal hematoma type 2 and/or thick subarachnoid hemorrhages (15.3\% vs. $5.1 \%, P=0.068)$ than did the non-tirofiban group ( $n=59)$. The tirofiban group compared to the non-tirofiban group achieved a better 3-month functional outcome (mRS score $0-2,52.5 \%$ vs. $32.2 \%, P=0.025) .{ }^{22}$ In another study, 98 patients who underwent thrombectomy followed by angioplasty, with or without stenting for the residual intracranial atherosclerotic stenosis, were divided into an IV tirofiban group $(n=30)$ and control group $(n=68)$. The rates of parenchymal hemorrhage (6.7\% [ $n=2]$ vs. $5.9 \%[n=4], P=0.99)$, symptomatic hemorrhage $(0 \%[n=0]$ vs. $2.9 \%[n=2], P=0.99), 90$-day mortality ( $0 \%[n=0]$ vs. $4.4 \%[n=3], P=0.55)$, and 90 -day $m R S$ score of $0-2(73.3 \%$ $[n=22]$ vs. $57.4 \%$ [ $n=39], P=0.13)$ were not significantly different between the tirofiban and control groups. Early reocclusion occurred less frequently in the tirofiban group than in the control group (3.3\% [n=1] vs. 25.0\% [n=17], $P=0.01)^{23}$

\section{Anticoagulants in patients with AIS with atrial fibrillation}

Long-term oral anticoagulation therapy with NOAC or warfarin is strongly recommended for secondary stroke prevention in patients with $\mathrm{AF}$ with prior ischemic stroke or TIA. ${ }^{11,13}$ During the acute period after AF-related ischemic stroke, anticoagulation might reduce the risk of recurrent ischemic stroke, but potentially increases the risk of $\mathrm{ICH}$.

Parenteral anticoagulation is not recommended because the benefit is offset by an increased risk of ICH. ${ }^{2,24-27}$ For oral anticoagulation, no clinical trial has formally investigated warfarin treatment in patients with AIS, and the large NOAC trials excluded AF patients who had experienced stroke within 7 to 30 days. ${ }^{28-31}$ Therefore, despite the clear benefit of long-term oral anticoagulation in AF patients with ischemic stroke or TIA, the optimal timing of initiating oral anticoagulation after acute cerebral ischemia is still uncertain. Recently, several observational studies and a small RCT have reported the safety and efficacy of early oral anticoagulation in AF patients with AIS or TIA. ${ }^{32-35}$ However, the evidence level from these studies is insufficient, and the recommendation for early oral anticoagulation is not unified across guidelines. The European, Canadian, and Australian stroke guidelines do not provide specific recommendations for the timing of initiating oral anticoagulation after AIS. ${ }^{7,812}$ The 2018 European Heart Rhythm Association provides specific recommendations for NOAC initiation according to stroke severity based on consensus of opinion. ${ }^{13}$ The 2019 American Stroke Association Guidelines state that it is reasonable to initiate oral anticoagulation between 4 and 14 days after the onset of neurological symptoms (moderate strength of recommendation based on evidence from non-randomized studies). ${ }^{6}$

\section{Observational studies}

The Early Recurrence and Cerebral Bleeding in Patients With Acute Ischemic Stroke and Atrial Fibrillation (RAF) study evaluated the risk of recurrent ischemic events and severe bleeding with anticoagulation regimens and the optimal timing for initiating anticoagulation. A total of 1,029 patients with AF with AIS were enrolled from 29 centers in European and Asian countries. ${ }^{32}$ The mean baseline NIHSS score was $9.2 \pm 7.3$, and it was lower in 766 patients treated with anticoagulation (oral anticoagulation alone, low molecular weight heparin [LMWH] alone, or bridging therapy [LMWH followed by oral anticoagulation]) than in 263 patients who did not receive any anticoagulation therapy. The rate of the composite outcome (stroke, TIA, symptomatic systemic embolism [SE], symptomatic cerebral bleeding, or major extracerebral bleeding) at 90 days was lowest in patients treated with oral anticoagulation alone (approximately $7 \%$ ), followed by bridging therapy (12.3\%), no anticoagulation (14.4\%), and LMWH alone (16.8\%). Anticoagulation initiated between 4 and 14 days from stroke onset compared with the other regimens combined was associated with a significant reduction in the composite outcome (adjusted HR, 
$0.53 ; 95 \% \mathrm{Cl}, 0.30$ to 0.93$)$ and ischemic events $(0.43 ; 95 \% \mathrm{Cl}$, 0.19 to 0.97 ), and a non-significant reduction in symptomatic intracranial bleeding $(0.39 ; 95 \% \mathrm{Cl}, 0.12$ to 1.19$)$. The analysis restricted to patients treated with oral anticoagulation alone also indicated that the best timing for initiating oral anticoagulation was 4 to 14 days after stroke onset.

The Clinical Relevance Of Microbleeds In Stroke-2 (CROMIS-2) study, a multicenter observation study from the United Kingdom and Netherlands, compared the 90-day clinical outcomes between 358 patients with early oral anticoagulation initiation ( 0 to 4 days after AIS or TIA) and 997 with late oral anticoagulation ( $\geq 5$ days or never started). ${ }^{36}$ The early group vs. the late group had a lower NIHSS score (median, 2 [interquartile range, IQR, 1 to 4] vs. 6 [IQR, 3 to 11]) and a higher proportion of NOAC use (46\% vs. $35 \%$ ). The rate of composite outcome (ischemic stroke, intracranial bleeding, TIA, or death) at 90 days was not significantly lower in the early group than in the late group (2\% vs. $5 \%$; adjusted $\mathrm{OR}, 0.85 ; 95 \% \mathrm{Cl}, 0.35$ to 2.08 ). Symptomatic intracranial bleeding occurred in two patients in the late group and none in the early group.

Stroke Acute Management with Urgent Risk-factor Assessment and Improvement-Non-Valvular Atrial Fibrillation (SAMURAI-NVAF), a Japanese multicenter observational study, compared the risks of stroke or SE and major bleeding between 475 patients who were treated with NOACs and 662 treated with warfarin during hospitalization for AIS. The median interval from stroke onset to initiating oral anticoagulant therapy was 3 days (IQR, 1 to 8) in the warfarin group and 4 days (IQR, 2 to 7$)$ in the NOAC group; the median NIHSS scores at admission were $11(I Q R, 4$ to 20$)$ and $4(I Q R, 1$ to 13), respectively. During 90 days, the NOAC group compared with the warfarin group had a similar risk of stroke or SE (2.84\% vs. 3.09\%; adjusted $\mathrm{HR}, 0.96 ; 95 \% \mathrm{Cl}, 0.44$ to 2.04 ) and a nonsignificant reduction in the occurrence of major bleeding (1.11\% vs. $2.61 \%$; adjusted $\mathrm{HR}, 0.63 ; 95 \% \mathrm{Cl}, 0.19$ to 1.78$).{ }^{33}$

The subsequent SAMURAI-NVAF study compared outcomes between 223 patients with early NOAC initiation ( 0 to 3 days after AIS or TIA, median 2 day [IQR, 1 to 3]) and 276 with late NOAC initiation ( $\geq 4$ days, median 6 days $[I Q R, 5$ to 9$]$ ). ${ }^{37}$ The early group had milder stroke severity and smaller infarct size that did the late group. The main analysis was conducted for 2-year event rates of stroke/SE, major bleeding, and death, which were comparable between the two groups. Of 90-day outcomes analyzed with a propensity score matched-cohort (193 patients in each group), the rate of stroke or SE was 4.2\% in the early group and $2.6 \%$ in the late group (adjusted $H R$, $1.49 ; 95 \% \mathrm{Cl}, 0.48$ to 4.64$)$. There were two deaths in each group, and $\mathrm{ICH}$ occurred in one patient in the late group and none in the early group.

Observational studies have reported the potential role of early NOAC use in patients with AF and AIS or TIA. However, these studies had limitations of indication bias and included mild stroke severity in the majority of patients.

\section{Clinical trial}

The Acute Stroke With Xarelto to Reduce Intracranial Hemorrhage, Recurrent Embolic Stroke, and Hospital Stay (Triple AXEL) was a randomized, multicenter, open-label, phase 2 trial that was conducted in Korea between April 2014 and December $2015{ }^{35} \mathrm{~A}$ total of $195 \mathrm{AF}-$ related AIS patients with mild severity within 5 days from symptom onset were randomized to rivaroxaban (10 mg daily for 5 days followed by 15 or $20 \mathrm{mg}$ daily) or warfarin (a target international normalized ratio of 2.0 to 3.0). The median interval from stroke onset to randomization was 2 days, and the median NIHSS score was 2.

The rivaroxaban and warfarin groups did not differ in the primary end point, a composite of new ischemic lesion or new ICH seen on follow-up magnetic resonance imaging at 4 weeks ( $49.5 \%$ vs. $54.5 \%$; relative risk [RR], $0.91 ; 95 \% \mathrm{Cl}, 0.69$ to 1.12 ; $P=0.49$ ). The two groups had comparable rates of new ischemic lesions (29.5\% vs. $34.5 \%$; RR, $0.83 ; 95 \% \mathrm{Cl}, 0.54$ to 1.26 ; $P=0.38)$ and new ICH (31.6\% vs. $28.7 \% ; \mathrm{RR}, 1.10 ; 95 \% \mathrm{Cl}, 0.70$ to $1.71 ; P=0.68)$. Each group had one clinical ischemic stroke related to $\mathrm{AF}$, and there was no symptomatic ICH. The trial had limitations of using an imaging surrogate marker endpoint and enrolling patients with mild stroke; however, it demonstrated that early oral anticoagulation within 5 days of stroke onset was safe and might be effective for preventing early clinical stroke recurrence in patients with mild AF-related AIS.

Currently, four RCTs are underway to compare early versus late initiation of NOAC in patients with AF-related AIS, which will enroll a total of 9,974 patients: 2,000 in the Early Versus Late Initiation of Direct Oral Anticoagulants in Post-ischaemic Stroke Patients with Atrial fibrillation (ELAN) trial, 3,474 in the OPtimal TIMing of Anticoagulation After Acute Ischaemic Stroke (OPTIMAS) trial, 3,000 in the Timing of oral anticoagulant therapy in acute ischemic stroke with atrial fibrillation (TIMING) trial, and 1,500 in the Optimal Delay Time to Initiate Anticoagulation After Ischemic Stroke in Atrial Fibrillation (START) trial. The results of these trials will guide healthcare providers to establish the optimal timing for early NOAC use in patients with AF and AIS.

\section{Consensus achievement}

We convened a panel of 35 expert neurologists from the KSS 
to reach a consensus with the modified Delphi method. The expert panel members were asked to provide their ratings for each recommendation on a 9-point scale (a score of 9 represented strong agreement and a score of 1 represented strong disagreement) modified from the Research AND Development Corporation method. ${ }^{38}$ Provided scores of 7-9 were considered as agreement, 4-6 as uncertainty, and 1-3 as disagreement. For each recommendation, if the agreement rate among experts was equal to or more than $75 \%$, we determined that the consensus was achieved. If the agreement rate was lower than $75 \%$, additional Delphi rounds were conducted with the revised recommendations reflecting the opinions of the panel members.

All 35 panel members provided their ratings (list of experts is in Appendix 1). In the first Delphi round, consensus was reached for three of the five revised recommendations. Revised recommendations for triple antiplatelet therapy (Question 2) and ticagrelor (Question 3) did not reach a consensus with an agreement rate of approximately 50\%, for which the writing group decided to exclude these recommendations in the current updated guidelines. During the first Delphi round, the need to recommend IV and/or intra-arterial use of glycoprotein IIb/ Illa receptor antagonists as rescue therapy during MT or emergent stenting was raised by several expert panel members.
Therefore, the writing group drafted additional recommendation for the use of glycoprotein Ilb/lla receptor antagonists in these conditions, which achieved a high agreement rate of $91.4 \%$ and accordingly is included in the updated guidelines. The agreement rates for individual recommendations are presented in Supplementary Table 1.

\section{Recommendations}

Updated recommendations for the use of antithrombotic agents in patients with AIS are summarized in Table 2 and Supplementary Table 2.

\section{Implications}

The current guideline update focused on antiplatelet therapy in minor AIS or high-risk TIA presenting within 24 hours from onset and early oral anticoagulation in AIS with AF. According to the current Clinical Research Collaboration for Stroke in Korea Statistics 2020 Report in Korea, 29.7\% of all patients with ischemic stroke arrived at a hospital within 24 hours of onset and had mild severity (NIHSS score $\leq 3$ ), and 7.4\% had AF and relatively mild stroke severity. ${ }^{39}$ Therefore, the revised recom-

Table 2. Summary of current recommendation

Comment

Antiplatelet agents

1. In the hemorrhage-excluded, acute ischemic stroke patients, the oral administration of aspirin should start within 24 to 48 No change hours of onset (the loading dose 160-300 mg) (LOE: la, GOR: A).

2. Aspirin cannot replace acute interventions including intravenous tPA (LOE: la, GOR: A).

3. For patients treated with intravenous thrombolysis, it is generally recommended to delay antithrombotic therapy up to 24 hours. However, when the benefit is expected to outweigh the risk, antithrombotic therapy may be initiated within 24 hours after intravenous tPA (LOE: III, GOR: B).

4. In general, intravenous glycoprotein Ilb/IIla receptor antagonists is not recommended in patients with acute ischemic stroke (LOE: Ib, GOR: A). However, intravenous and/or intra-arterial use of glycoprotein Ilb/Illa receptor antagonists can be considered in highly selected patients who require rescue therapy during mechanical thrombectomy or emergent angioplasty/stenting, taking into account benefit and risk (LOE: IV, GOR: C).

5. In patients presenting with acute minor ischemic stroke (NIHSS score $0-3$ ) or high-risk TIA $\left(A_{B C D}\right.$ score $\left.\geq 4\right)$, dual antiplatelet therapy with aspirin and clopidogrel initiated within 24 hours from the onset and maintained for up to 21-30 days is recommended to further reduce the risk of early recurrent stroke and major ischemic event (LOE: la, GOR: A).

Anticoagulants

1. There is no scientific evidence on the usefulness of heparin used within 48 hours of ischemic cerebral infarction. It might increase the risk of bleeding, compared with aspirin (LOE: la, GOR: A).

2. LMWH or heparinoids is not recommended as an early treatment of cerebral infarction (LOE: la, GOR: A).

3. Use of anticoagulants within 24 hours of intravenous tPA administration is not recommended (LOE: Ila, GOR: B).

4. For patients with acute ischemic stroke and atrial fibrillation, it is recommended to start oral anticoagulation when the risk New recommendation of hemorrhagic transformation is expected to be low. It may be reasonable to start oral anticoagulation between 4 and 14 days after stroke onset. However, in patients with a high risk of recurrent stroke and low risk of hemorrhagic transformation, oral anticoagulation might be initiated within 5 days from stroke onset (LOE: III, GOR: B).

LOE, level of evidence; GOR, grade of recommendations; tPA, tissue plasminogen activator; NIHSS, National Institutes of Health Stroke Scale; TIA, transient ischemic attack; LMWH, low molecular weight heparin.
No change

Revised from the previous recommendation

Revised from the previous recommendation

New recommendation

No change

No change

No change 
mendations would significantly influence the management of at least one-third of patients with AIS or TIA who are at high risk of early stroke progression or recurrence.

\section{Supplementary materials}

Supplementary materials related to this article can be found online at https://doi.org/10.5853/jos.2021.02628.

\section{Disclosure}

The authors have no financial conflicts of interest.

\section{References}

1. CAST (Chinese Acute Stroke Trial) Collaborative Group. CAST: randomised placebo-controlled trial of early aspirin use in 20,000 patients with acute ischaemic stroke. Lancet 1997; 349:1641-1649.

2. International Stroke Trial Collaborative Group. The International Stroke Trial (IST): a randomised trial of aspirin, subcutaneous heparin, both, or neither among 19435 patients with acute ischaemic stroke. Lancet 1997;349:1569-1581.

3. Chen ZM, Sandercock P, Pan HC, Counsell C, Collins R, Liu LS, et al. Indications for early aspirin use in acute ischemic stroke: a combined analysis of 40000 randomized patients from the Chinese acute stroke trial and the international stroke trial. On behalf of the CAST and IST collaborative groups. Stroke 2000;31:1240-1249.

4. Wang Y, Wang Y, Zhao X, Liu L, Wang D, Wang C, et al. Clopidogrel with aspirin in acute minor stroke or transient ischemic attack. N Engl J Med 2013;369:11-19.

5. Johnston SC, Easton JD, Farrant M, Barsan W, Conwit RA, Elm JJ, et al. Clopidogrel and aspirin in acute ischemic stroke and high-risk TIA. N Engl J Med 2018;379:215-225.

6. Powers WJ, Rabinstein AA, Ackerson T, Adeoye OM, Bambakidis NC, Becker K, et al. Guidelines for the early management of patients with acute ischemic stroke: 2019 update to the 2018 guidelines for the early management of acute ischemic stroke: a guideline for healthcare professionals from the American Heart Association/American Stroke Association. Stroke 2019;50:e344-e418.

7. Boulanger JM, Lindsay MP, Gubitz G, Smith EE, Stotts G, Foley $\mathrm{N}_{\text {, et }}$ al. Canadian stroke best practice recommendations for acute stroke management: prehospital, emergency department, and acute inpatient stroke care, 6th edition, update 2018. Int J Stroke 2018;13:949-984.

8. Australian clinical guidelines for stroke management. Clinical guidelines for stroke management. Chapter 3: Acute medical and surgical management. Stroke Foundation. https://informme.org.au/guidelines/clinical-guidelines-for-stroke-management. 2022. Accessed January 17, 2022.

9. Bath PM, Woodhouse $\sqcup$, Appleton JP, Beridze M, Christensen $\mathrm{H}$, Dineen RA, et al. Antiplatelet therapy with aspirin, clopidogrel, and dipyridamole versus clopidogrel alone or aspirin and dipyridamole in patients with acute cerebral ischaemia (TARDIS): a randomised, open-label, phase 3 superiority trial. Lancet 2018;391:850-859.

10. Johnston SC, Amarenco P, Albers GW, Denison H, Easton JD, Evans SR, et al. Ticagrelor versus aspirin in acute stroke or transient ischemic attack. N Engl J Med 2016;375:35-43.

11. Powers WJ, Rabinstein AA, Ackerson T, Adeoye OM, Bambakidis NC, Becker K, et al. 2018 Guidelines for the early management of patients with acute ischemic stroke: a guideline for healthcare professionals from the American Heart Association/American Stroke Association. Stroke 2018;49:e46-e110.

12. Klijn CJ, Paciaroni $M$, Berge $E_{1}$ Korompoki $E_{1}$ Kõrv J, Lal $A$, et al. Antithrombotic treatment for secondary prevention of stroke and other thromboembolic events in patients with stroke or transient ischemic attack and non-valvular atrial fibrillation: a European Stroke Organisation guideline. Eur Stroke J 2019;4:198-223.

13. Steffel J, Verhamme P, Potpara TS, Albaladejo P, Antz M, Desteghe L, et al. The 2018 European Heart Rhythm Association practical guide on the use of non-vitamin $\mathrm{K}$ antagonist oral anticoagulants in patients with atrial fibrillation. Eur Heart J 2018;39:1330-1393.

14. The writing group of clinical practice guideline for stroke. Clinical Practice Guideline for Stroke. Seoul, KR: Clinical Research Center for Stroke, 2009.

15. United States Department of Health and Human Services; Agency for Health Care Policy and Research. Acute Pain Management: Operative or Medical Procedures and Trauma. Rockville, MD: U.S. Dept. of Health and Human Services, Public Health Service, Agency for Health Care Policy and Research, 1993;107.

16. Pan $Y$, Elm JJ, Li H, Easton JD, Wang Y, Farrant $M$, et al. Outcomes associated with clopidogrel-aspirin use in minor stroke or transient ischemic attack: a pooled analysis of Clopidogrel in High-Risk Patients With Acute Non-Disabling Cerebrovascular Events (CHANCE) and Platelet-Oriented Inhibition in New TIA and Minor Ischemic Stroke (POINT) trials. JAMA Neurol 2019;76:1466-1473.

17. Johnston SC, Amarenco P, Denison H, Evans SR, Himmelmann $A$, James $S$, et al. Ticagrelor and aspirin or aspirin alone 
in acute ischemic stroke or TIA. N Engl J Med 2020;383:207217.

18. Jeong HG, Kim BJ, Yang MH, Han MK, Bae HJ, Lee SH. Stroke outcomes with use of antithrombotics within 24 hours after recanalization treatment. Neurology 2016;87:996-1002.

19. Lee YS, Bae HJ, Kang DW, Lee SH, Yu K, Park JM, et al. Cilostazol in Acute Ischemic Stroke Treatment (CAIST Trial): a randomized double-blind non-inferiority trial. Cerebrovasc Dis 2011;32:65-71.

20. Adams HP Jr, Effron MB, Torner J, Dávalos A, Frayne J, Teal P, et al. Emergency administration of abciximab for treatment of patients with acute ischemic stroke: results of an international phase III trial: Abciximab in Emergency Treatment of Stroke Trial (AbESTT-II). Stroke 2008;39:87-99.

21. Zhang S, Hao Y, Tian X, Zi W, Wang H, Yang D, et al. Safety of intra-arterial tirofiban administration in ischemic stroke patients after unsuccessful mechanical thrombectomy. J Vasc Interv Radio/ 2019;30:141-147.

22. Kim YW, Sohn SI, Yoo J, Hong JH, Kim CH, Kang DH, et al. Local tirofiban infusion for remnant stenosis in large vessel occlusion: tirofiban ASSIST study. BMC Neurol 2020;20:284.

23. Baek BH, Yoon W, Lee YY, Kim SK, Kim JT, Park MS. Intravenous tirofiban infusion after angioplasty and stenting in intracranial atherosclerotic stenosis-related stroke. Stroke 2021;52:1601-1608.

24. Saxena R, Lewis S, Berge E, Sandercock PA, Koudstaal PJ. Risk of early death and recurrent stroke and effect of heparin in 3169 patients with acute ischemic stroke and atrial fibrillation in the International Stroke Trial. Stroke 2001;32:2333-2337.

25. Berge $E$, Abdelnoor M, Nakstad PH, Sandset PM. Low molecular-weight heparin versus aspirin in patients with acute ischaemic stroke and atrial fibrillation: a double-blind randomised study. HAEST Study Group. Heparin in Acute Embolic Stroke Trial. Lancet 2000;355:1205-1210.

26. Paciaroni M, Agnelli G, Micheli S, Caso V. Efficacy and safety of anticoagulant treatment in acute cardioembolic stroke: a meta-analysis of randomized controlled trials. Stroke 2007; 38:423-430.

27. Whiteley WN, Adams HP Jr, Bath PM, Berge $E_{1}$ Sandset PM, Dennis $M$, et al. Targeted use of heparin, heparinoids, or low-molecular-weight heparin to improve outcome after acute ischaemic stroke: an individual patient data meta-analysis of randomised controlled trials. Lancet Neurol 2013;12: 539-545.

28. Giugliano RP, Ruff CT, Braunwald E, Murphy SA, Wiviott SD, Halperin $J L_{\text {, et }}$ al. Edoxaban versus warfarin in patients with atrial fibrillation. N Engl J Med 2013;369:2093-2104.

29. Patel MR, Mahaffey KW, Garg J, Pan G, Singer DE, Hacke W, et al. Rivaroxaban versus warfarin in nonvalvular atrial fibrillation. N Engl J Med 2011;365:883-891.

30. Granger CB, Alexander JH, McMurray JJ, Lopes RD, Hylek EM, Hanna $M$, et al. Apixaban versus warfarin in patients with atrial fibrillation. N Engl J Med 2011;365:981-992.

31. Connolly SJ, Ezekowitz MD, Yusuf S, Eikelboom J, Oldgren J, Parekh $A$, et al. Dabigatran versus warfarin in patients with atrial fibrillation. N Engl J Med 2009;361:1139-1151.

32. Paciaroni M, Agnelli G, Falocci N, Caso V, Becattini C, Marcheselli $S$, et al. Early recurrence and cerebral bleeding in patients with acute ischemic stroke and atrial fibrillation: effect of anticoagulation and its timing: the RAF Study. Stroke 2015;46:2175-2182.

33. Arihiro $S_{1}$ Todo $K_{1}$ Koga $M$, Furui $E_{1}$ Kinoshita $N$, Kimura $K_{1}$ et al. Three-month risk-benefit profile of anticoagulation after stroke with atrial fibrillation: the SAMURAI-Nonvalvular Atrial Fibrillation (NVAF) study. Int J Stroke 2016;11:565-574.

34. Seiffge DJ, Traenka C, Polymeris A, Hert L, Peters N, Lyrer $P$, et al. Early start of DOAC after ischemic stroke: risk of intracranial hemorrhage and recurrent events. Neurology 2016;87: 1856-1862.

35. Hong KS, Kwon SU, Lee SH, Lee JS, Kim YJ, Song TJ, et al. Rivaroxaban vs warfarin sodium in the ultra-early period after atrial fibrillation-related mild ischemic stroke: a randomized clinical trial. JAMA Neurol 2017;74:1206-1215.

36. Wilson D, Ambler G, Banerjee G, Shakeshaft C, Cohen $H$, Yousry $T A$, et al. Early versus late anticoagulation for ischaemic stroke associated with atrial fibrillation: multicentre cohort study. J Neurol Neurosurg Psychiatry 2019;90:320325.

37. Mizoguchi T, Tanaka K, Toyoda K, Yoshimura S, Itabashi R, Takagi $M$, et al. Early initiation of direct oral anticoagulants after onset of stroke and short- and long-term outcomes of patients with nonvalvular atrial fibrillation. Stroke 2020;51: 883-891.

38. Brook RH, Chassin MR, Fink A, Solomon DH, Kosecoff J, Park RE. A method for the detailed assessment of the appropriateness of medical technologies. Int J Technol Assess Health Care 1986;2:53-63.

39. Clinical research collaboration for stroke in Korea: CRCS-K statistics 2020 report. Korean Stroke Registry. http://www. Strokedb.or.kr/report/index.Asp. 2020. Accessed December $17,2021$. 
Appendix 1. List of responding participants

\begin{tabular}{|c|c|}
\hline Participants & Affiliation \\
\hline Ja Seong Koo & Department of Neurology, Seoul St. Mary's Hospital, The Catholic University of Korea College of Medicine, Seoul, Korea \\
\hline Joon-Tae Kim & Department of Neurology, Chonnam National University Hospital, Chonnam National University Medical School, Gwangju, Korea \\
\hline Jong-Moo Park & Department of Neurology, Uijeongbu Eulji Medical Center, Eulji University, Uijeongbu, Korea \\
\hline Jay Chol Choi & Department of Neurology, Jeju National University Hospital, Jeju National University School of Medicine, Jeju, Korea \\
\hline Jae-Kwan Cha & Department of Neurology, Dong-A University Hospital, Dong-A University College of Medicine, Busan, Korea \\
\hline Kyung-Yul Lee & Department of Neurology, Gangnam Severance Hospital, Yonsei University College of Medicine, Seoul, Korea \\
\hline Sang Won Han & Department of Neurology, Inje University Sanggye Paik Hospital, Inje University College of Medicine, Seoul, Korea \\
\hline Kyung-Ho Yu & Department of Neurology, Hallym University Sacred Heart Hospital, Hallym University College of Medicine, Anyang, Korea \\
\hline Kwang-Yeol Park & Department of Neurology, Chung-Ang University Hospital, Chung-Ang University College of Medicine, Seoul, Korea \\
\hline Soo Joo Lee & Department of Neurology, Eulji University Hospital, Eulji University, Daejeon, Korea \\
\hline Kyung Bok Lee & Department of Neurology, Soonchunhyang University Seoul Hospital, Soonchunhyang University College of Medicine, Seoul, Korea \\
\hline Jong-Ho Park & Department of Neurology, Myongji Hospital, Hanyang University College of Medicine, Goyang, Korea \\
\hline Yong-Seok Lee & $\begin{array}{l}\text { Department of Neurology, Seoul Metropolitan Government-Seoul National University Boramae Medical Center, Seoul National } \\
\text { University College of Medicine, Seoul, Korea }\end{array}$ \\
\hline Hyo Suk Nam & Department of Neurology, Severance Stroke Center, Severance Hospital, Yonsei University College of Medicine, Seoul, Korea \\
\hline Man-Seok Park & Department of Neurology, Chonnam National University Hospital, Chonnam National University Medical School, Gwangju, Korea \\
\hline Gyeong-Moon Kim & Department of Neurology and Stroke Center, Samsung Medical Center, Sungkyunkwan University School of Medicine, Seoul, Korea \\
\hline Sung-II Sohn & Department of Neurology, Keimyung University Dongsan Hospital, Keimyung University School of Medicine, Daegu, Korea \\
\hline Ju-Hun Lee & Department of Neurology, Kangdong Sacred Heart Hospital, Hallym University College of Medicine, Seoul, South Korea \\
\hline Jun Lee & Department of Neurology, Yeungnam University Medical Center, Yeungnam University College of Medicine, Daegu, Korea \\
\hline Sung Hyuk Heo & Department of Neurology, Kyung Hee University Medical Center, Kyung Hee University College of Medicine, Seoul, Republic of Korea \\
\hline Keun-Hwa Jung & Department of Neurology, Seoul National University Hospital, Seoul National University College of Medicine, Seoul, Korea \\
\hline Jei Kim & Department of Neurology, Chungnam National University Hospital, Chungnam National University School of Medicine, Daejeon, Korea \\
\hline Eung-Gyu Kim & Department of Neurology, Inje University Busan Paik Hospital, Inje University College of Medicine, Busan, Korea \\
\hline Hahn Young Kim & Department of Neurology, Konkuk University Hospital, Konkuk University School of Medicine, Seoul, Korea \\
\hline Ji Man Hong & Department of Neurology, Ajou University School of Medicine, Ajou University Medical Center, Suwon, Korea \\
\hline Keun-Sik Hong & Department of Neurology, Inje University Ilsan Paik Hospital, Inje University College of Medicine, Goyang, Korea \\
\hline Sang-Bae Ko & Department of Neurology, Seoul National University Hospital, Seoul National University College of Medicine, Seoul, Korea \\
\hline Hong-Kyun Park & Department of Neurology, Inje University Ilsan Paik Hospital, Inje University College of Medicine, Goyang, Korea \\
\hline Ji Hoe Heo & Department of Neurology, Severance Stroke Center, Severance Hospital, Yonsei University College of Medicine, Seoul, Korea \\
\hline Joung-Ho Rha & Department of Neurology, Inha University Hospital, Inha University College of Medicine, Incheon, Korea \\
\hline Sun U. Kwon & Department of Neurology, Asan Medical Center, University of Ulsan College of Medicine, Seoul, Korea \\
\hline Hee-Joon Bae & $\begin{array}{l}\text { Department of Neurology and Cerebrovascular Center, Seoul National University Bundang Hospital, Seoul National University } \\
\text { College of Medicine, Seongnam, Korea }\end{array}$ \\
\hline Jong S. Kim & Department of Neurology, Asan Medical Center, University of Ulsan College of Medicine, Seoul, Korea \\
\hline Byung-Woo Yoon & Department of Neurology, Uijeongbu Eulji Medical Center, Eulji University, Uijeongbu, Korea \\
\hline Byung-Chul Lee & Department of Neurology, Hallym University Sacred Heart Hospital, Hallym University College of Medicine, Anyang, Korea \\
\hline
\end{tabular}


Supplemental Table 1. Results of Delphi Consensus

\begin{tabular}{|c|c|c|c|c|}
\hline Questions and recommendations & $\begin{array}{l}\text { Delphi round } \\
\text { achieving consensus }\end{array}$ & $\begin{array}{c}\text { Agreement } \\
\text { (score 7-9) (\%) }\end{array}$ & $\begin{array}{l}\text { Uncertainty } \\
\text { (score 4-6) (\%) }\end{array}$ & $\begin{array}{l}\text { Disagreement } \\
(\text { score } 1-3)(\%)\end{array}$ \\
\hline \multicolumn{5}{|l|}{ 2.3.4 Antiplatelet agents } \\
\hline $\begin{array}{l}\text { Q1-1. Do you think that the recommendation of dual antiplatelet therapy in minor } \\
\text { ischemic stroke or high-risk TIA patients is necessary to be added in Korean stroke } \\
\text { guidelines based on the results of recent clinical trials (CHANCE and POINT)? }\end{array}$ & First round & 97.1 & 0.0 & 2.9 \\
\hline $\begin{array}{l}\text { Q1-2. If yes, do you agree with this new recommendation? } \\
\text { Recommendation } 5 \text {. In patients presenting with acute minor ischemic stroke } \\
\text { (NIHSS score } 0-3) \text { or high-risk TIA }\left(A B C D_{2} \text { score } \geq 4\right) \text {, dual antiplatelet therapy } \\
\text { with aspirin and clopidogrel initiated within } 24 \text { hours from the onset and } \\
\text { maintained for up to } 21-30 \text { days is recommended to further reduce the risk of } \\
\text { early recurrent stroke and major ischemic event (LOE: la, GOR: A). }\end{array}$ & First round & 80.0 & 14.7 & 2.9 \\
\hline $\begin{array}{l}\text { 02-1. Is it necessary to add a new recommendation of triple antiplatelet therapy in } \\
\text { patients with acute ischemic stroke patients? }\end{array}$ & First round & 51.4 & 17.1 & 31.4 \\
\hline $\begin{array}{l}\text { 02-2. If yes, do you agree with this new recommendation? } \\
\text { Recommendation } 6 \text {. In patients presenting with acute ischemic stroke or TIA, } \\
\text { triple antiplatelet therapy with a combination of aspirin, clopidogrel, and } \\
\text { dipyridamole for the first } 1 \text { month is not recommended (LOE: Ib, GOR: A). }\end{array}$ & First round & 51.4 & 0.0 & 0.0 \\
\hline $\begin{array}{l}\text { 03-1. Do you think that the recommendation whether to use ticagrelor for acute } \\
\text { ischemic stroke patients is necessary to be included in the current guideline? }\end{array}$ & First round & 51.4 & 14.3 & 34.3 \\
\hline $\begin{array}{l}\text { 03-2. If yes, do you agree with this new recommendation? } \\
\text { Recommendation 7. Ticagrelor is not recommended over aspirin in patients with } \\
\text { acute ischemic stroke or TIA until more data become available (LOE: Ib, GOR: A). }\end{array}$ & First round & 51.4 & 0.0 & 0.0 \\
\hline $\begin{array}{l}\text { Q4-1. Do you think that the recommendation of avoidance of aspirin within } 24 \text { hours } \\
\text { after intravenous thrombolysis needs to be revised? } \\
\text { Recommendation } 3 \text {. Aspirin should not be taken within } 24 \text { hours of thrombolysis } \\
\text { (LOE: la, GOR: A). }\end{array}$ & First round & 88.6 & 8.6 & 2.9 \\
\hline Q4-2. If yes, do you agree with the revised recommendation? & First round & 88.6 & 0.0 & 0.0 \\
\hline
\end{tabular}

Recommendation 3. For patients treated with intravenous thrombolysis, it is generally recommended to delay antithrombotic therapy up to 24 hours. However, when the benefit is expected to outweigh the risk, antithrombotic therapy may be initiated within 24 hours after intravenous thrombolysis (LOE: III, GOR: B).

05. Do you have any idea about other recommendations on antiplatelet therapy in the acute phase of ischemic stroke patients?

1. In the hemorrhage-excluded, acute ischemic stroke patients, the oral administration of aspirin should start within 24 to 48 hours of onset (the loading dose 160-300 mg) (LOE: la, GOR: A).

2. Aspirin cannot replace acute interventions including IVT (LOE: la, GOR: A).

4. Intravenous injection of the glycoprotein Ill/Illa receptor antagonists, including abciximab, is not recommended in patients with acute ischemic stroke (LOE: Ib, GOR: A).

2.3.5 Anticoagulants

Q6-1. Do you think that the recommendation of early initiation of anticoagulants in AFrelated ischemic stroke patients could be added?

06-2. If yes, do you agree with this new recommendation? Recommendation 4. For patients with acute ischemic stroke and atrial fibrillation, it is recommended to start oral anticoagulation when the risk of hemorrhagic transformation is expected to be low. It may be reasonable to start oral anticoagulation between 4 and 14 days after stroke onset. However, in patients with high risk of recurrent stroke and low risk of hemorrhagic transformation, oral anticoagulation might be initiated within 5 days from stroke onset (LOE: III, GOR: B).

07. Do you have any idea about other recommendations on anticoagulant therapy in the acute phase of ischemic stroke patients?

1. There is no scientific evidence on the usefulness of heparin used within 48 hours of ischemic cerebral infarction. It might increase the risk of bleeding, compared with aspirin (LOE: la, GOR: A).

2. LMWH or heparinoids is not recommended as an early treatment of cerebral infarction (LOE: Ia, GOR: A).

3. Use of anticoagulants within 24 hours of rt-PA administration is not recommended (LOE: Ila, GOR: B).

No new opinion

No new opinion

Second round

First round 88.5

First round

No new opinion

Q, question; TIA, transient ischemic attack; CHANCE, The Clopidogrel in High-Risk Patients with Acute Nondisabling Cerebrovascular Events; POINT, The Platelet-Oriented Inhibition in New TIA and Minor Ischemic Stroke; NIHSS, National Institutes of Health Stroke Scale; LOE, level of evidence; GOR, grade of recommendation; IVT, intravenous thrombolysis; AF, atrial fibrillation; LMWH, low molecular weighted heparin; rt-PA, recombinant tissue plasminogen activator. 
Supplemental Table 2. Comparison of changes in recommendations between previous and revised guidelines

Previous guideline in 2009

Antiplatelet agents 3 . Aspirin should not be taken within 24 hours of thrombolysis (LOE: la, GOR: A).

4. Intravenous injection of the glycoprotein IIb/ Illa receptor antagonists, including abciximab, is not recommended in patients with acute ischemic stroke (LOE: Ib, GOR: A).

Anticoagulants
Updated guideline in 2022

3. For patients treated with intravenous thrombolysis, it is generally recommended to delay antithrombotic therapy up to 24 hours. However, when the benefit is expected to outweigh the risk, antithrombotic therapy may be initiated within 24 hours after intravenous tPA (LOE: III, GOR: B).

4. In general, intravenous glycoprotein IIb/IIla receptor antagonists is not recommended in patients with acute ischemic stroke (LOE: Ib, GOR: A). However, intravenous and/or intra-arterial use of glycoprotein Ilb/Illa receptor antagonists can be considered in highly selected patients who require rescue therapy during mechanical thrombectomy or emergent angioplasty/stenting, taking into account benefit and risk (LOE: IV, GOR: C).

5. In patients presenting with acute minor ischemic stroke (NIHSS score 0-3) or high-risk TIA $\left(A B C D_{2}\right.$ score $\left.\geq 4\right)$, dual antiplatelet therapy with aspirin and clopidogrel initiated within 24 hours from the onset and maintained for up to 21-30 days is recommended to further reduce the risk of early recurrent stroke and major ischemic event (LOE: la, GOR: A).

4. For patients with acute ischemic stroke and atrial fibrillation, it is recommended to start oral anticoagulation when the risk of hemorrhagic transformation is expected to be low. It may be reasonable to start oral anticoagulation between 4 and 14 days after stroke onset. However, in patients with high risk of recurrent stroke and low risk of hemorrhagic transformation, oral anticoagulation might be initiated within 5 days from stroke onset (LOE: III, GOR: B).

LOE, level of evidence; GOR, grade of recommendation; tPA, tissue plasminogen activator; NIHSS, National Institutes of Health Stroke Scale; TIA, transient ischemic attack. 